\title{
Peptic ulcer bleeding: accessory risk factors and interactions with non-steroidal anti-inflammatory drugs
}

J Weil, M J S Langman, P Wainwright, D H Lawson, M Rawlins, R F A Logan, T P Brown, M P Vessey, M Murphy, D G Colin-Jones

\begin{abstract}
Aims-To determine risk factors for peptic ulcer bleeding other than non-steroidal anti-inflammatory drugs (NSAIDs).

Methods-Data on possible antecedent risk factors obtained in a large case control study of 1121 patients admitted to hospitals in Glasgow, Newcastle, Nottingham, Oxford, and Portsmouth with bleeding peptic ulcers were compared with the same information obtained in 989 population controls. Data were analysed by logistic regression with the calculation of odds ratios $(O R)$ and $95 \%$ confidence intervals (CI).

Results-From a logistic regression model, oral anticoagulants (OR $7.8 ; 95 \%$ CI 2.8-21.5), previous peptic ulcer (3.8; 2.6-4.9), treatment for heart failure (5.9; 2.3-13.1), oral corticosteroid use $(2.7 ; 1.3-$ $4.5)$, treatment for diabetes $(3.1 ; 1.2-4.3)$, and current smoking $(1.6 ; 1.2-2.0)$ were all independent risk factors. No association was found with use of calcium channel antagonists. Odds ratios for concomitant NSAID usage were multiplicative with the exception of current smoking.

Conclusions-Some $45 \%$ of admissions for peptic ulcer bleeding in England and Wales in those aged 60 or more are calculated to be attributable to, or associated with, these accessory risk factors, which, together with those associated with aspirin or other NSAID use will account for over $80 \%$ of predisposing factors to ulcer bleeding.

(Gut 2000;46:27-31)
\end{abstract}

Keywords: peptic ulcer; risk factors; anti-inflammatory drugs

There have been many reports on the risks of hospitalisation for peptic ulcer disease or its complications associated with the use of different non-steroidal anti-inflammatory drugs (NSAIDs). ${ }^{1-12}$ However, less is known about other factors and how they interact with NSAIDs in altering the chances of ulcer complications; and this predominantly stems from a paucity of studies with population controls, hospital controls being obviously inappropriate. We have previously reported on overall odds ratios of 3.8 (3.1-4.5) for non-aspirin NSAID use in the three months prior to admission ${ }^{6}$ and $3.2(2.3-4.4)$ for daily aspirin usage of at least one month's duration. ${ }^{12}$
These studies included large numbers of population controls, and we have now undertaken detailed analyses of our data with regard to concurrent illness and medication, previous peptic ulcer or dyspepsia, as well as smoking habits and alcohol consumption.

\section{Patients and methods}

These have been fully described elsewhere. ${ }^{6}$ Briefly, 1121 patients aged 60 and over, admitted to hospitals in Glasgow, Newcastle, Nottingham, Oxford, and Portsmouth between April 1986 and January 1991, with haematemesis or melaena due to gastric or duodenal ulceration, were studied. Each patient was matched for age and sex with a hospital control taken from the acute medical intake, and a community control taken from the same general practice as the patient. Patients and controls were only included once. In each centre patients and controls were interviewed by the same research assistant using a structured questionnaire. This included detailed information on previous NSAID and analgesic intake. Current smoking habits and alcohol intake were also included in the inquiry. In addition information was included on the use of other medications in the week prior to admission. These were initially classified into 27 distinct categories using groupings employed by the British National Formulary. Reported drug intake was checked against general practitioner (GP) records.

Odds ratios (OR), 95\% confidence intervals (CI), and interactions of specific factors with NSAIDs (both aspirin and non-aspirin), were calculated using logistic regression with SAS software. The following were included as independent variables: a previous history of peptic ulcer disease; a history of dyspepsia lasting longer than one week in the past year; NSAID use; current smoking; and alcohol intake. Other risk factors were also included as necessary, and to correct for the greater comorbidity in the patients, a variable for two or more associated illnesses (other than peptic ulcer) was included. Underlying illness was defined both as self reported and by prescribed medication. Thus heart failure was considered both as self reported, and when both digoxin and diuretics were taken; and diabetes was considered as self reported, and again when oral hypoglycaemics or insulin were in use. Statistical significance

Abbreviations used in this paper: NSAID, non-steroidal anti-inflammatory drug. 
Table 1 Comparison of patients and controls

\begin{tabular}{|c|c|c|}
\hline & $\begin{array}{l}\text { Patients } \\
(n=1121)\end{array}$ & $\begin{array}{l}\text { Community controls } \\
(n=989)\end{array}$ \\
\hline \multicolumn{3}{|l|}{ Current illnesses } \\
\hline Heart failure & 65 & 9 \\
\hline Ischaemic heart disease & 116 & 107 \\
\hline Other cardiovascular & 221 & 197 \\
\hline Respiratory disease & 183 & 130 \\
\hline Diabetes & 69 & 27 \\
\hline Other endocrine & 46 & 33 \\
\hline Previous peptic ulcer & 290 & 89 \\
\hline Other gastrointestinal & 98 & 106 \\
\hline Neurological disease & 114 & 76 \\
\hline Haematological disease & 36 & 27 \\
\hline Other diseases & 188 & 161 \\
\hline Two or more illnesses $\star$ & 438 & 256 \\
\hline Nursing home & 37 & 3 \\
\hline \multicolumn{3}{|l|}{ Drug use in the previous week } \\
\hline Antacids & 252 & 109 \\
\hline Antiulcer & 167 & 54 \\
\hline Antibiotics & 66 & 29 \\
\hline Digoxin & 81 & 38 \\
\hline Diuretics & 379 & 237 \\
\hline Potassium supplements & 90 & 42 \\
\hline Antianginals/antiarrythmics & 257 & 218 \\
\hline Oral corticosteroids & 64 & 20 \\
\hline Bronchodilators & 117 & 94 \\
\hline Warfarin & 39 & 7 \\
\hline Psychotropics & 176 & 111 \\
\hline Three or more drugs & 451 & 237 \\
\hline \multicolumn{3}{|l|}{ Other } \\
\hline Alcohol consumption $>7$ units/week & 197 & 145 \\
\hline Current smokers $\dagger$ & 324 & 220 \\
\hline Dyspepsia in the past year $>1$ week & 588 & 259 \\
\hline
\end{tabular}

^Excluding peptic ulcer disease.

†As defined by at least one cigarette a day, one cigar a week, or 1 ounce of pipe tobacco a month.

tests for comparison of logistic regression models were based on the difference between -2 log likelihood scores. The referent groups chosen were patients and community controls not exposed to each risk factor. The hospital controls, being inappropriate, were excluded from consideration.

\section{Results}

Table 1 shows the number of patients and community controls with recorded current illnesses or medical treatments, these being grouped in broad systematic categories. $\mathrm{Pa}-$ tients were generally more likely to suffer from a wide variety of current illnesses than randomly chosen community controls. As might be expected, there was broad congruence between self reported patterns of illness and drug treatments in use.

Table 2 presents the odds ratios with $95 \%$ of confidence intervals for significant associations detected. All of these were independent. The raised odds ratios associated with oral corticosteroid use did not differ materially for gastric (OR 2.73; 95\% CI 1.34-5.53) and duodenal ulcer $(2.11 ; 1.02-4.35)$. Addition of rheumatoid arthritis to the logistic regression as a separate factor did not alter odds ratios obtained here or elsewhere.

Odds ratios were also raised significantly in association with warfarin treatment, and independently with heart failure as categorised by concurrent treatment with digoxin and diuretics. Separate examination of possible associations with antianginal and antiarrhythmic treatments showed no significant differences, and examination of risks associated with calcium channel antagonist use gave an odds ratio of $0.96(95 \%$ CI $0.62-1.48)$ with $6.0 \%$ of patients and $6.1 \%$ of controls reporting use in
Table 2 Odds ratios (OR) for significant accessory risk factors compared with community controls

\begin{tabular}{lll}
\hline Category & OR & $95 \% C I$ \\
\hline Oral corticosteroids & 2.7 & $1.3-4.5$ \\
Warfarin & 7.8 & $2.8-21.5$ \\
Previous peptic ulcer & 3.8 & $2.6-4.9$ \\
Dyspepsia in past year & 4.0 & $3.1-4.8$ \\
Heart failure & 5.9 & $2.3-13.1$ \\
Diabetes & 3.1 & $1.2-4.3$ \\
Current smoking & 1.6 & $1.2-2.0$ \\
\hline
\end{tabular}

Odds ratios determined by unconditional logistic regression model.

CI, confidence interval.

Table 3 Interaction between oral corticosteroid and non-steroidal anti-inflammatory drug (NSAID) use

\begin{tabular}{lllll}
\hline & & & $\begin{array}{l}\text { Community } \\
\text { NSAID }\end{array}$ & Corticosteroid \\
$(n=1121)$ & $\begin{array}{l}\text { Ontrols } \\
(n=989)\end{array}$ & $\begin{array}{l}\text { Odds } \\
\text { ratio }\end{array}$ \\
\hline- & - & 431 & 643 & $1.0^{\star}$ \\
+ & - & 626 & 326 & 3.8 \\
- & + & 26 & 14 & 2.6 \\
+ & + & 38 & 6 & 9.0 \\
\hline
\end{tabular}

${ }^{\star}$ Referent.

Table 4 Non-steroidal anti-inflammatory drug (NSAID) use stratified by current smoking status

\begin{tabular}{|c|c|c|c|c|}
\hline NSAID & $\begin{array}{l}\text { Current } \\
\text { smoker }\end{array}$ & $\begin{array}{l}\text { Patients } \\
(n=1111) *\end{array}$ & $\begin{array}{l}\text { Community } \\
\text { controls } \\
(n=989)\end{array}$ & OR $(95 \% C I)$ \\
\hline - & - & 312 & 541 & $1.0 \dagger$ \\
\hline+ & - & 477 & 228 & $3.6(2.9-4.5)$ \\
\hline - & + & 159 & 149 & $1.9(1.4-2.4)$ \\
\hline+ & + & 163 & 71 & $4.0(2.9-5.5)$ \\
\hline
\end{tabular}

*Smoking status unknown for 10 patients.

†Referent.

OR, odds ratio; CI, confidence interval.

the previous week. Risks associated with diabetes did not appear to differ for those receiving oral treatments, insulin, or dietary advice alone. Other categories of drugs, such as antiepileptics or bronchodilators, showed no significant associations (defined as odds ratios of more than 2.0 with probability values greater than 0.02).

Current smoking, a history of prior diagnosed peptic ulcer, and a history of dyspepsia in the past year were associated with significantly raised risk (table 2), but alcohol consumption was not, the odds ratio being 1.20 (95\% CI 0.88-1.79).

Table 3 shows the joint effect of NSAIDs and oral corticosteroids using non-takers of either drug as the referent. Odds ratios were approximately tripled for corticosteroid or NSAID use alone, and increased by 10 -fold when both were taken together. Logistic regression analysis showed similar multiplicative effects with NSAIDs for the other accessory risk factors, with the exception of current smoking.

Table 4 shows the separate and joint effects associated with current smoking and with NSAID use. The calculated risk for smokers who take NSAIDs is little different from the figure for NSAID takers who are nonsmokers. 


\section{Discussion}

We have analysed the data from our study population to determine risk factors other than NSAIDs for peptic ulcer bleeding. The data for hospital controls are not included as hospital controls came from the acute medical intake and form an inherently sick population. Including them in the analysis of second diseases or their treatments as risk factors would have been illogical. By the same token, the use of hospital controls, whether inpatient or outpatient, is subject to unquantifiable biases which make judgement of the true weight of observed differences impossible. Although comparisons between patients with ulcer complications and population controls are clearly preferable, the operation of Berkson's bias in the patient group with a second disease being intrinsically more likely to be reported in hospital attendees ${ }^{13}$ than nonattendees could still pose problems. Given the serious nature of bleeding from peptic ulcer, and the lack of any reason to suspect that such patients with associated illnesses are more likely to be admitted on clinical grounds, we think that bias through the preferential admission of patients with two diseases is unlikely.

Our data suggest that apart from a previous history of ulcer or dyspepsia, the occurrence of heart failure or diabetes as well as smoking and oral anticoagulant intake are significant accessory risk factors. We think it unlikely that information bias could account for these results. Interview times and subjects' cooperation were recorded and were not significantly different between the two groups. Recall bias is possible because current illness was self reported. For patients hospital notes were also available as potential interviewer prompts. However, for medication taken in the previous week bias is less likely as the medicines would be to hand in the home. Drug histories were also checked against GP records and found to be in substantial agreement. ${ }^{6}$ Bias due to non-response in sick controls seems unlikely because control responses were high (86\%), and associations detected with disease outside the gut were selective.

Diabetes and heart failure remained significant risk factors for peptic ulcer bleeding after adjustment for other underlying illnesses and other known risk factors. We know of no comparable data for diabetes. Silverstein and colleagues ${ }^{14}$ found a weakly significant association between a history of heart disease and serious NSAID related upper gastrointestinal complications, a result consonant with data indicating that patients with coronary artery stents who receive both prophylactic aspirin and warfarin are at high risk of gastrointestinal bleeding compared with those receiving aspirin alone. ${ }^{15}$ This appears to be the only dataset which bears on the plausible hypothesis that anticoagulant use poses material risks. One previous claim ${ }^{16}$ has been of an association with calcium channel antagonist use, albeit of modest degree, with a second result showing a non-significantly raised odds ratio of $1.2 .{ }^{17}$ Our data, in agreement with others, ${ }^{18}$ do not confirm this. Taken overall it seems unlikely that calcium channel antagonists pose risks. The associations we have detected with heart failure and diabetes could have arisen by chance in multiple comparative analyses, but this is unlikely because the odds ratios are reasonably substantial, and because the numbers on which estimates are based are large. Possible mechanisms include mucosal congestion or slowed hepatic drug metabolism in heart failure, and vascular disease impairing mucosal integrity in diabetes.

The association between corticosteroid treatment and ulcer disease has been variously described as a myth, ${ }^{19}$ associated with a twofold increase in risk, ${ }^{20}$ and most recently as only present when steroids are prescribed together with NSAIDs. ${ }^{9}$ In a meta-analysis of 42 randomised studies of non-complicated ulcer, Conn and Blitzer ${ }^{19}$ found no increase in risk and concluded that the association dated from anecdotal reports during the early use of ACTH and adrenocorticosteroids. In a larger meta-analysis of 71 randomised studies, Messer and colleagues ${ }^{20}$ showed a relative risk for peptic ulcer haemorrhage of 2.1 (1.1-4.0) and concluded that the earlier study had failed to show an association because of small numbers, and differences in datasets included and excluded. A case control study of computerised Medicaid files ${ }^{9}$ later gave a relative risk for hospitalisation for peptic ulcer of 2.0 (1.3-3.0). The risk was increased only in those who concurrently received NSAIDs. It was concluded that steroids may be more important in delaying healing of lesions caused by NSAIDs than in causing de novo ulceration. It is unclear why steroids should only delay the healing of NSAID induced ulcers and we believe our finding of an increased overall risk with multiplicative effects for concomitant NSAID administration is more plausible.

Alcohol consumption has long been believed to increase the chances of peptic ulcer bleeding but there is little evidence from the endoscopic era when duodenal as well as gastric ulcer could be confidently recognised. Henry et al found an odds ratio of 6.0 for alcohol consumption, but the comparator group appears to have been a predominantly hospital control group, ${ }^{4}$ and so the conclusion is questionable. One much smaller study of ours in the past suggested no interaction with alcohol consumption, ${ }^{21}$ while a second more recent study suggested that risk was raised by threefold with heavy alcohol consumption (over $80 \mathrm{~g}$ per day in men and $60 \mathrm{~g}$ per day in women). ${ }^{22}$ Our questionnaire was only able to distinguish unsafe levels ( $>21$ units a week) of beer consumption, and grouped together all subjects drinking seven units a week or more of wine or spirits. Neither high levels of beer consumption nor drinking seven units of alcohol a week or more were significant risk factors. It may be argued that if a population of heavy alcohol consumers could be identified then a raised risk might be demonstrable; 
Table 5 Attributable risk associated with individual risk factors

\begin{tabular}{lr}
\hline & \multicolumn{1}{l}{$n$} \\
\hline Aspirin or non-aspirin NSAID use & 3300 \\
Smoking & 1000 \\
Oral anticoagulants & 300 \\
Treated heart failure & 475 \\
Treated diabetes & 425 \\
Oral corticosteroids & 325 \\
Prior peptic ulcer & 1900 \\
Total $^{\star}$ & 7725 \\
\hline
\end{tabular}

$\star 77 \%$ of the total 10000 cases estimated to occur each year in those aged 60 years and over.

NSAID, non-steroidal anti-inflammatory drug.

however, it appears that heavy alcohol consumption was not characteristic of the relatively elderly group we studied, who, in general terms, form two thirds of those admitted to hospital with ulcer bleeding in the United Kingdom.

Evidence conflicts as to whether smoking is, ${ }^{22}$ or is not ${ }^{21}$ an accessory factor. Previous studies have been small, and are therefore likely to be unreliable. We have, however, shown here that smoking is a weak risk factor for peptic ulcer bleeding. The lack of an apparent interaction with NSAID intake may reflect the comparative weakness of the smoking association, but raised fibrinogen levels associated with smoking ${ }^{23}$ could tend to protect against an interaction increasing the risk of bleeding. Our comparator group seems likely to be representative because their level of smoking was equivalent to the $21 \%$ found in a similar age group as part of The Health Survey for England. ${ }^{24}$

Previous data, based on smaller numbers of patients, again conflict in suggesting that a history of ulcer does, ${ }^{22}$ or does not ${ }^{21}$ increase the risk of ulcer bleeding. Both these series were much smaller than ours. A further study is unreliable because the comparator was a hospital control. ${ }^{7}$ Our results suggest that risks may be substantial, whether measured as prior history of ulcer, or as recent dyspeptic symptoms. Our data give information about the likely influence of Helicobacter pylori infection through any association with previous ulcer disease, but do not help directly to determine the role of $H$ pylori without ulcer.

The population attributable risk (PAR) of disease depends not only on the relative risk (for which the odds ratios in these studies are close approximations), but also on the extent of exposure in the population, and from a public health perspective is at least as important as the relative risk. We have previously calculated ${ }^{6}$ that about a third of an estimated 10000 annual hospitalisations in England and Wales for peptic ulcer bleeding in those aged 60 and over are attributable to NSAID or aspirin use. Assuming that our patient and community control populations are representative we can use the data on potential risk factors in table 1 and the odds ratios in table 2 (these being adjusted for the simultaneous operation of other factors) to estimate the numbers of cases of ulcer bleeding associated with accessory risk factors.
Thus $25.9 \%$ (290) of patients had a history of previous peptic ulcer compared with $9.0 \%$ (91) of 989 community controls with an odds ratio adjusted for the operation of other factors of 3.8. Episodes associated with prior ulcer would therefore have been expected in $6.9 \%$ of patients, giving an aetiological fraction of 0.19 . In the same way, the aetiological fractions associated with smoking, heart failure, diabetes, use of oral corticosteroids, and of anticoagulants can be calculated as 0.1, 0.05, $0.04,0.03$, and 0.03 respectively to give a total of 0.44 . To these can be added 0.22 for prior NSAID use ${ }^{6}$ and 0.11 for prophylactic aspirin use, $^{12}$ to give an overall total of 0.77 . The remaining $20-25 \%$ has to accommodate all other known and unknown risk factors, including short term aspirin use and $H$ pylori infection without ulcer. This gives some support to the contested claim that $H$ pylori infection does not adversely affect the course of NSAID associated ulcer ${ }^{25-27}$ because the amount of potentially unexplained disease appears limited (table 5).

This research was funded by the Medical Research Council. We are grateful to our research associates for data collection.

1 Garcia-Rodriguez LA, Walker AM, Perez-Gutenhann S Non-steroidal anti-inflammatory drugs and gastrointestinal hospitalization in Saskatchewan: a cohort study. Epidemiology 1992;3:337-42.

2 Garcia Rodriguez LA, Jick H. Risk of upper gastrointestinal bleeding and perforation associated with individual nonsteroidal anti-inflammatory drugs. Lancet 1994;343:76972 .

3 Griffin MR, Piper JM, Daugherty JR, et al. Non steroidal anti-inflammatory drug use and increased risk for peptic
ulcer disease in elderly persons. Ann Intern Med 1991;114: ulcer disease

4 Henry D, Dobson A, Turner C. Variability in the risk of major gastrointestinal complications from non aspirin non steroidal anti-inflammatory drugs. Gastroenterology 1993; 105:1978-88.

5 Kaufman DW, Kelly JP, Sheehan JE, et al. Non-steroidal anti-inflammatory drug use in relation to major upper gastrointestinal bleeding. Clin Pharmacol Ther 1993;53: 485-94.

6 Langman MJS, Weil J, Wainwright P, et al. Risks of bleeding peptic ulcer associated with individual non-steroidal anti-inflammatory drugs. Lancet 1994;343:1075-9.

7 Laporte JR, Carne X, Vidal X, et al. Upper gastrointestinal bleeding in relation to previous use of analgesics and bleeding in relation to previous use of analgesics and
non-steroidal anti-inflammatory drugs. Lancet 1991;337: 85-9.

8 Nobili A, Mosconi P, Franzosi MG, et al. Non-steroidal anti-inflammatory drugs and upper gastrointestinal bleeding, a postmarketing surveillance case-control study. Pharmacoepidemiol Drug Safety 1992;1:65-72.

9 Piper JM, Ray WA, Daugherty JR, et al. Corticosteroid use and peptic ulcer disease: role of non steroidal antiinflammatory drugs. Ann Intern Med 1991;114:735-40.

10 Savage RL, Moller PW, Ballantyne CL, et al. Variation in the risk of peptic ulcer complications with non-steroidal anti-inflammatory drug therapy. Arthritis Rheum 1993;36: $84-90$.

11 Somerville K, Faulkner G, Langman MJS. Non-steroidal anti-inflammatory drugs and bleeding peptic ulcer. Lancet 1986;i:452-4.

12 Weil J, Colin-Jones D, Langman M, et al. Prophylactic aspirin and risk of peptic ulcer bleeding. BMF 1995;310:827-

13 Berkson J. Limitations of the application of 4-fold tables to hospital data. Biometrics Bulletin 1946;2:47-53.

14 Silverstein FE, Graham DY, Senior JR, et al. Misoprostol reduces serious gastrointestinal complications in patients with rheumatoid arthritis receiving non-steroidal antiinflammatory drugs. Ann Intern Med 1995;123:241-9.

15 Younossi ZM, Strum WB, Schatz RA, et al. Effects of combined anticoagulation and low-dose aspirin treatment on upper gastrointestinal bleeding. Dig Dis Sci 1997;42: $79-82$.

16 Pahor M, Guralnik JM, Furberg CD, et al. Risk of gastrointestinal haemorrhage with calcium antagonists in hypertensive patients over 67 years old. Lancet 1996:347: 1061-5.

17 Kelly JP, Laszlo A, Kaufman DW, et al. Major upper gastrointestinal bleeding and the use of calcium channel blockers. Lancet 1999;353:559. 
18 Smalley WE, Ray WA, Daugherty JR, et al. No association between calcium channel blocker use and confirmed bleeding peptic ulcer disease. Am f Epidemiol 1998;148: $350-4$

19 Conn HO, Blitzer BL. Non-association of adrenocorticosteroid therapy and peptic ulcer. N Engl f Med 1976;294: 473-9.

20 Messer J, Reitman D, Sacks HS, et al. Association of adrenocorticosteroid therapy and peptic ulcer. $N$ Engl $\mathcal{F}$ Med 1983;309:21-4

21 Somerville KW, Faulkner G, Langman MJS. Risk factors for non-steroidal drug-associated upper gastrointestinal bleeding. Eur f Gastroenterol Hepatol 1992;4:645-9.

22 Hochhain P, Berkelmans I, Czerichow P, et al. Which patients taking non-aspirin non-steroidal antiinflammatory drugs bleed? A case-control study. Eur $\mathcal{f}$ Gastroenterol Hepatol 1995;7:419-26.
23 Meade TW, Roderick PJ, Brennan PJ, et al. Extra-cranial bleeding and other symptoms due to low dose aspirin and bleeding and other symptoms due to low dose aspirin and 68: $1-6$.

24 Office of Population Censuses and Surveys. Health Survey for England 1992. London: HMSO, 1994.

25 Cullen DJE, Hawkey GM, Greenwood DC, et al. Peptic ulcer bleeding in the elderly: relative roles of Helicobacter pylori and non-steroidal anti-inflammatory drugs. Gut 1997;41:459-62.

26 Hawkey CJ, Tulassey Z, Szczepanski L, et al. Randomised controlled trial of Helicobacter pylori eradication in patients on non-steroidal anti-inflammatory drugs: HELP NSAIDs study. Lancet 1998;352:1016-21.

27 Chan FKL, Sung JJY, Chung SCS, et al. Randomised controlled trial of eradication of Helicobacter pylori before non-steroidal anti-inflammatory drug therapy to prevent peptic ulcers. Lancet 1997;350:975-9. 